\title{
Differences in Cardiovascular Morbidity and Mortality between Previously Known and Newly Diagnosed Adult Diabetics
}

\author{
J. B. Herman, J.H. Medalie, and U. Goldbourt \\ Hadassah University Hospital, Jerusalem and Tel Aviv University Medical School, Israel
}

Summary. 498 diabetics were discovered in a survey of ischaemic heart disease in 10,059 men aged 40 years and over in Israel. - The diabetics were divided into previously known and newly diagnosed. - During a 5 year follow-up period, the incidence rates of fatal and non-fatal myocardial infarction and the incidence of intermittent claudication were considerably greater in both groups of diabetics than in non-diabetics. The frequency of hypertension and left ventricular hypertrophy was about the same in both groups, and in non-diabetics. - However, the pattern of increased morbidity for angina pectoris and the increased rate of sudden death is seen only in the previously diagnosed diabetics. Different pathological mechanisms might be responsible for angina pectoris and sudden death, as compared to myocardial infarction and these might be related in diabetics to the duration and severity of the disease.

Key words: Prospective study, ischaemic heart disease, diabetics.

Clinical [1, 2, 3] and pathological [4] studies testify to the frequency of cardiovascular $(\mathrm{CV})$ complications in diabetes, particularly since the increased longevity following the introduction of insulin and antibiotics. Almost 20 years after the introduction of the oral hypoglycaemic agents this still holds true [5]. Epidemiological surveys have confirmed a higher prevalence [6] and incidence [7] of cardiovascular disease (CVD) amongst diabetics and in association with hyperglycaemia [6].

In a long term prospective ischaemic heart disease study, a recent analysis of the factors related to cardiovascular disease [8], showed diabetes to be significanctly related to the incidence of cardiovascular disease in Israel. The present paper deals, in greater detail, with the cardiovascular data from the diabetics discovered in the above study.

The diabetics have been divided into two groups: those previously diagnosed and those diagnosed for the first time at the survey. We report here the five year incidence of cardiovascular disease in the two groups of diabetics in relation to the rest of the survey population (the controls) found to be free of these cardiovascular abnormalities on entry to the study.

\section{Subjects and Methods}

\section{The Study Population}

The study population, described in detail elsewhere [9], consisted of a stratified random sample of Israeli male permanent civil service and municipal employees aged 40 years and over, and included at least 1000 men from six regions of birth: Eastern Europe, Central Europe, Southern Europe, Israel, the Middle East and North Africa. Because of the nature of governmental responsibility in Israel many kinds of occupational employment are represented, ranging from highly responsible administration posts through professional and clerical positions to simple unskilled labour tasks. A total of 10,059 men, $86.2 \%$ of those invited, were examined in the first survey in 1963. Two further surveys were carried out at 2 and 3 year intervals respectively; close to $98 \%$ of the living subjects were re-examined in the third survey, five years after the initial survey, and 100 percent of the subjects were accounted for. 


\section{Survey Data}

The survey data included a medical and demographic history, anthropometric and physical examination and a 12 lead E.C.G. at rest. In addition, results were obtained from biochemical tests as well as dietary and psychosocial questionnaires.

\section{Identification and Criteria for Diagnosis of Diabetics}

Diabetics in the study were identified as follows: all those with a casual blood glucose value of $130 \mathrm{mg}$ or over (Somogyi-Nelsen (S-N)) and/or a history of diabetes were considered suspect diabetics. Thereafter, for each suspect diabetic, additional data were collected from the personal physician or the specialist at the diabetes clinics. Where data available were inadequate for the diagnosis of diabetes by our criteria, the subject was asked to report to the survey laboratory for a glucose tolerance test (GTT), after a carbohydrate-rich diet for at least 3 days. $50 \mathrm{~g}$ glucose in water was ingested after a twelve hour fast.

Details of the criteria for the diagnosis of diabetes are given in a previous publication [10]. Briefly, the diagnosis of diabetes was based either on the finding of abnormal fasting blood glucose tests, generally three, above $130 \mathrm{mg} / 100 \mathrm{ml}$. Hagedorn-Jensen $(\mathrm{H}-\mathrm{J})$ method, or, where a GTT was performed, by a score of 1 point or more on the following scale:

\begin{tabular}{|c|c|c|c|}
\hline \multicolumn{4}{|c|}{$\begin{array}{l}\mathrm{H}-\mathrm{J} \\
(\mathrm{mg} / 100 \mathrm{ml})\end{array}$} \\
\hline Fasting level & $\geqslant 120$ & $\geqslant 100$ & $1 / 2$ point \\
\hline 1 hour level & $\geqslant 200$ & $\geqslant 170$ & $t_{i 2}$ point \\
\hline 2 hour level & $\geqslant 140$ & $\geqslant 120$ & ${ }_{12}^{1}$ point \\
\hline
\end{tabular}

498 of the study population $(4.95 \%)$ were found to be diabetic at the first prevalence survey. These were divided into two groups according to whether diabetes had been previously diagnosed prior to the survey ("Clinical" diabetics, $\mathrm{n}=296$ ) or newly diagnosed (Survey diabetics, $n=202$ ). The data on which the diagnosis of diabetes for the two groups was based is shown below.

\begin{tabular}{lcc}
\hline & $\begin{array}{c}\text { Previously diagnosed } \\
\text { ("Clinical") diabetics }\end{array}$ & $\begin{array}{l}\text { Newly diagnosed } \\
\text { (Survey) diabetics }\end{array}$ \\
\hline $\begin{array}{l}\text { Glucose tolerance } \\
\text { Other data }\end{array}$ & 51 & 159 \\
\hline Total & 245 & 43 \\
\hline
\end{tabular}

In $78.7 \%$ of newly diagnosed diabetics the diagnosis was based on the results of a GTT, whereas in $82.8 \%$ of the previously diagnosed diabetics, be- cause of the high fasting blood glucose values, the diagnosis of diabetes was based on data other than the GTT.

\section{Diagnosis of Cardiovascular Disease}

Myocardial infarction (M.I.) was examined in two ways: 1. By E.C.G. diagnosis only: an E.C.G. interpretation of probable or possible infarct, based on criteria described in the appendix of reference [11].

2. "Group I Ischaemic heart disease" (IHD I) as described elsewhere [11]. This was composed of a majority of cases of verified history of myocardial infarction and included cases of E.C.G. diagnosis of possible infarct or myocardial ischaemia associated with definite angina pectoris.

Angina Pectoris (A.P.): The diagnosis of angina pectoris was based on criteria recommended by the World Health Organization [12]. A "closed followup" type of questionnaire was used. Diagnosis of angina pectoris depended on the site, duration, precipitating and relieving factors of chest pain [13].

Intermittent Claudication (I.C.): Pain in one or both calves brought on by walking and relieved by rest within ten minutes.

Hypertension: In this paper, a mercury sphygmomanometer reading of 160 or over systolic and/ or a diastolic reading of 95 or over, in men in whom the blood pressure was $<140 / 90$ in the initial survey 5 years previously.

Left ventricular hypertrophy (L.V.H.) was based on E. C. G. diagnosis [11].

Cause-specific mortality data were obtained as described elsewhere [14].

\section{Statistical Analysis}

Differences between incidence of morbidity (or mortality) in previously (clinically) diagnosed vs. newly (survey) diagnosed diabetics were analysed by $\chi^{2}$ test with one degree of freedom in an effort to reject the hypothesis that the same morbidity (or mortality) force acted in both groups. Significance of the $\mathrm{O} / \mathrm{E}$ (observed/expected values) ratio is given as the significance of the "proportion comparison" [15] viz. incidence in the diabetic group compared to incidence among non-diabetics. Expected incidence figures are those obtained when the non-diabetic rates were applied to a diabetic group. 
Table 1. The mortality ratio in diabetics for cardiovascular and other causes during a 5 year incidence study

\begin{tabular}{|c|c|c|c|c|c|c|c|c|c|c|c|c|}
\hline & \multicolumn{4}{|c|}{ Previously Diagnosed Diabetics } & \multicolumn{4}{|c|}{ Newly Diagnosed Diabetics } & \multicolumn{4}{|c|}{ Total Diabetics } \\
\hline & Number & $\overline{\mathrm{Obs}}$ & $\operatorname{Exp}^{a}$ & $\mathrm{O} / \mathrm{E}$ ratio & Number $^{b}$ & Obs & $\operatorname{Exp}^{\mathrm{a}}$ & $\overline{\mathrm{O} / \mathrm{E} \text { ratio }}$ & Number & Obs & $\operatorname{Exp}^{a}$ & $\mathrm{O} / \mathrm{E}$ ratio \\
\hline $\begin{array}{l}\text { Myocardial Infarction } \\
\text { Presumed Myocardial }\end{array}$ & 270 & 12 & 3.5 & 3.4 & 209 & 8 & 2.4 & 3.4 & 479 & 20 & 5.9 & 3.4 \\
\hline Infarction (Death $<1 \mathrm{~h}$ ) & 270 & 8 & 2.2 & 3.6 & 209 & 1 & 1.6 & $(0.6)$ & 479 & 9 & 3.8 & 2.4 \\
\hline All causes & 270 & 40 & 17.1 & 2.3 & 209 & 16 & 11.4 & 1.4 & 479 & 56 & 28.5 & 2.0 \\
\hline
\end{tabular}

() Based on small numbers

a Expected values are according to rates among the non-diabetic population at first survey

b Includes diabetics classified as "unknown" at first survey

Table 2. Cardiovascular morbidity ratio in diabetes (based on a 5-year incidence study). For abbreviations see legend to Figure 2

\begin{tabular}{|c|c|c|c|c|c|c|c|c|c|c|c|c|}
\hline & \multicolumn{4}{|c|}{ Previously Diagnosed Diabetics } & \multicolumn{4}{|c|}{ Newly Diagnosed Diabetics } & \multicolumn{4}{|c|}{ Total Diabetics } \\
\hline & Num & Obs & $\operatorname{Exp}^{a}$ & O/E ratio & Num & er Obs & $\operatorname{Exp}^{2}$ & $\mathrm{O} / \mathrm{E}$ ratio & Total & Obs & $\operatorname{Exp}^{2}$ & $\mathrm{O} / \mathrm{E}$ ratio \\
\hline \multicolumn{13}{|l|}{ Myocardial Infarction } \\
\hline I. H.D. I & 197 & 18 & 6.0 & 3.0 & 172 & 9 & 4.9 & 1.8 & 369 & 27 & 10.9 & 2.5 \\
\hline A.P. only & 181 & 18 & 5.6 & 3.2 & 166 & 3 & 5.0 & $(0.6)$ & 347 & 21 & 10.6 & 2.0 \\
\hline I. C. & 221 & 20 & 9.3 & 2.2 & 179 & 17 & 7.1 & 2.3 & 400 & 37 & 16.4 & 2.3 \\
\hline $\begin{array}{l}\text { Hypertension } \\
\text { L. V. H. only }\end{array}$ & 118 & 5 & 5.3 & 1.0 & 75 & 4 & 3.1 & $(1.3)$ & 193 & 9 & 8.4 & 1.1 \\
\hline (E.C.G. diagnosis) & 212 & 19 & 20.2 & 0.9 & 173 & 15 & 15.7 & 0.9 & 385 & 34 & 35.9 & 1.0 \\
\hline
\end{tabular}

a Expected numbers are according to the rates among the non-diabetic population

() Small numbers $=$ exp. 5.0 or less

\section{Results}

Mortality from all causes was 56 observed versus 28 expected among all 1963 diabetics. Of these, 40 (vs 17 expected) occurred among previously known diabetics (Table 1).

Approximately half the deaths were cardiovascular deaths. There was a three and a half fold greater mortality from myocardial infarction (M. I.) in both groups of diabetics $(\mathrm{p}<0.01)$ (Fig. 1).

The morbidity for four groups of cardiovascular disease states e.g. (a) Myocardial infarction by E. C. G. (b) Ischaemic heart disease, group I (IHD group I). (c) Angina pectoris (A.P.) only, and (d) Intermittent claudication (I.C.) is considerably greater in the total diabetics than in non-diabetics (Table 2). The higher observed/expected morbidity ratio is more evident in the previously diagnosed (or clinically diagnosed) diabetics, reaching three times that of non-diabetics for Group I Ischaemic Heart Disease ("IHD I") $(p<0.005)$ as well as for angina pectoris (A.P.) ( $\mathrm{p}<0.005)$. Newly diagnosed diabetics might also have higher rates except for angina pectoris. The incidence of angina pectoris in previously diagnosed diabetics (clinical diabetics) was five times that of the survey-diagnosed diabetics $(\mathrm{p}<0.005)$. Hypertension and left ventricular hypertrophy ("L. V.H. - E.C.G.") diagnosis show no incidence differences between both groups of

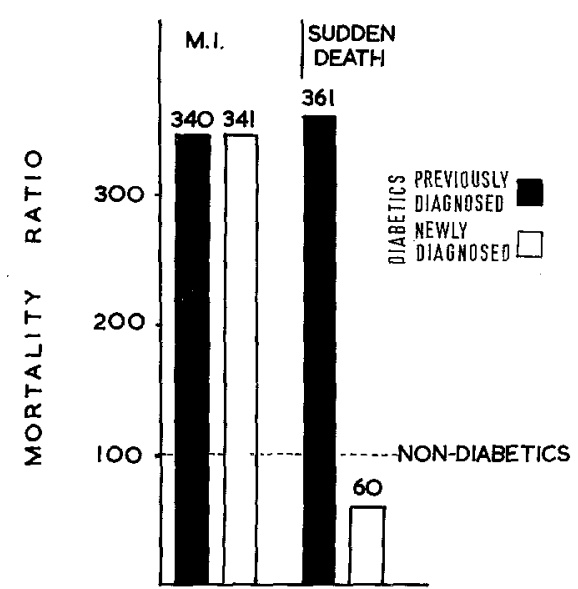

Fig. 1. Differences in cardiovascular mortality between previously diagnosed and newly diagnosed diabetics -5 year incidence. The mortality "ratio" is the ratio of the number of cases observed to the number of cases expected, multiplied by 100 to produce whole numbers. - The number at the top of each column indicates this ratio. Absolute numbers of events are included in Table 1. Abbreviations: M. I. = Myocardial infarction

diabetics or the non-diabetics. The differences in cardiovascular mortality and morbidity in the two groups of diabetics are shown diagrammatically in Figures 1 and 2 as a "ratio" of the number of cases observed to the number of cases expected. This fraction was multiplied by 100 to produce whole numbers. 


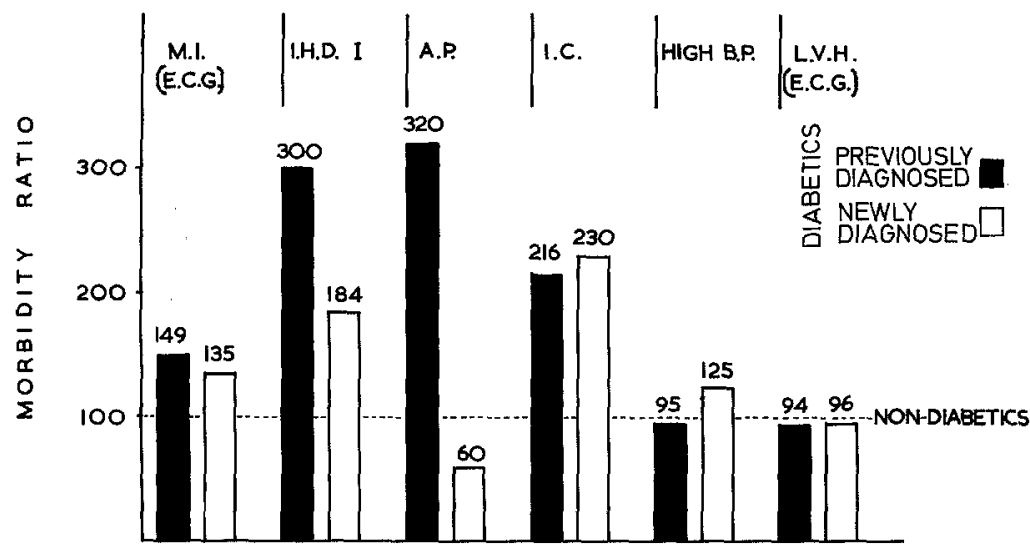

Fig. 2. Differences in cardiovascular morbidity between previously diagnosed and newly diagnosed diabetics -5 year incidence. The morbidity "ratio" is the ratio of the number of cases observed to the number of cases expected, multiplied by 100 to produce whole numbers. The number at the top of each column indicates this ratio. Absolute numbers of cpisodes are included in Table 2. Abbreviations: M. I. (E. C. G.) = Myocardial infarction (E. C. G. diagnosed) - I. H.D. I = Ischaemic heart discase, Group I - A.P. = Angina pectoris - L. V.H. = Left ventricular hypertrophy - B.P. $=$ Blood pressure

Table 3. Distribution of risk factors for cardiovascular disease in two groups of diabetics

\begin{tabular}{llllllr}
\hline $\begin{array}{l}\text { Age-adjusted } \\
\text { mean values }\end{array}$ & $\begin{array}{l}\text { Cholesterol } \\
(\mathrm{mg} / 100 \mathrm{ml})\end{array}$ & $\begin{array}{l}\text { Systolic } \\
\text { B.P. }(\mathrm{mm})\end{array}$ & $\begin{array}{l}\text { Diastolic } \\
\text { B.P. }(\mathrm{mm})\end{array}$ & $\begin{array}{l}\mathrm{W} / \mathrm{H}^{\mathrm{a}} \\
\left(\mathrm{gm} / \mathrm{cm}^{2}\right)\end{array}$ & $\begin{array}{l}\text { Uric acid } \\
(\mathrm{mg} / 100 \mathrm{ml})\end{array}$ & $\begin{array}{l}\% \text { above } \\
\mathrm{B} . \mathrm{P} .160 / 95\end{array}$ \\
\hline Previously known & 217 & 138 & 84 & 2.61 & 4.21 & $8.4 \%$ \\
Newly diagnosed & 220 & 146 & 87 & 2.70 & 4.56 & $13.6 \%$ \\
Total population & 209 & 135 & 84 & 2.57 & 4.75 & $8.5 \%$ \\
\hline
\end{tabular}

a Quetelet's index

\section{Associated Cardiovascular Risk Factors}

Some of the well recognised risk factors are given in Table 3 for the newly and previously diagnosed diabetics. Mean cholesterol values, W/ $\mathrm{H}^{2}$ (obesity index) and blood pressure were higher in the newly diagnosed than in the previously diagnosed diabetics. Mean uric acid was lower in diabetics than in the normals, being lower in previously diagnosed than in newly diagnosed diabetics [10]. The table shows that previously diagnosed diabetics do not incur higher coronary heart rates due to an unfavourable "risk profile".

\section{Discussion}

The cardiovascular morbidity and mortality of diabetics is higher than that of non-diabetics, in keeping with other population studies $[6,7,16]$. The pattern of cardiovascular disease is similar in the two groups of diabetics with two possible exceptions.

One is almost certain: the morbidity for angina pectoris in previously diagnosed diabetics is about three times that of non-diabetics, but it is less than expected amongst the newly diagnosed diabetics. With respect to sudden death from presumed myocardial infarction there is a hint of a similar behaviour in diabetics, but numbers are too small for definite conclusions.
It has been widely accepted that hypertension occurs with increased frequency in diabetics $[6,17$, 18]; however, others [19] have refuted a higher frequency of hypertension in diabetes. Our data show that the incidence of new cases of hypertension in men found to be normotensive (BP $<140 / 90) 5$ years previously is no higher in diabetics than in non-diabetics. There was also no increased incidence of left ventricular hypertrophy (by E.C. G.).

The reasons for the difference in angina pectoris incidence in the two groups of diabetics are not clear. Patients with previously unrecognised diabetes (i. e. the newly diagnosed) may be those who fail to communicate their symptoms to the doctor. This could account for a somewhat lower incidence of angina pectoris in survey diagnosed diabetics, but does not seem likely; if such a reason were operative, one might have expected a similarly lower incidence of intermittent claudication in newly diagnosed diabetics.

It is possible that the differences in cardiovascular disease incidence noted may be emphasised by the lesser severity [16] and shorter duration [1] of diabetes in the newly diagnosed group. Our previously diagnosed diabetics, in whom the vast majority had raised fasting blood glucose values and therefore considerably raised values at other times of a glucose tolerance test, are comparable to frank diabetics. Eighty percent of the newly diagnosed diabetics, on the other hand, were chemical dia- 
betics and would correspond with the "borderline group" of Keen et al. [16], for whom a cardiovascular morbidity prevalence intermediate between that of frank diabetics and non-diabetics was described. The validity of the diagnosis of diabetes on a constant scale of GTT criteria may be questioned since glucose tolerance has been shown to decrease with age [20]. However, we have shown [10] that diabetics diagnosed by the criteria used at our surveys have characteristics similar to those of clinically diagnosed diabetics with regard to cholesterol, obesity and uric acid level.

A detailed analysis of the cardiovascular risk factors in various groups of diabetics in the study is to be published. An analysis of some of the cardiovascular risk factors shows that newly diagnosed diabetics in fact had an unfavourable coronary heart disease profile, which certainly cannot explain their reduced coronary heart disease incidence as compared to that of the previously known diabetics.

Of the different components of the lipids, serum cholesterol seems to have the greatest predictive value for cardiovascular disease [21]. Mean cholesterol values were higher in the diabetics than in the non-diabetics and were higher in the newly diagnosed than in the previously diagnosed diabetics. In keeping with this, there were more diabetics than non-diabetics with cholesterol values above the 80th centile and more newly diagnosed than previously diagnosed. This same pattern applied also for the $\mathrm{W} / \mathrm{H}^{2}$ (obesity) index. The lower cholesterol and obesity index values in previously known diabetics might be due to the effects of treatment or to the diabetes itself. With regard to uric acid we have shown that there is an inverse relationship between serum uric acid and diabetes. Uric acid is lower in previously diagnosed than in newly diagnosed diabetics [10]. Uric acid amongst the group who subsequently develop diabetes, though, is higher than amongst non-diabetics [22], a factor which may be relevant to cardiovascular disease [23]. We have no data for triglycerides, nor, at this stage, for cigarette smoking in the two groups.

Our studies have shown that the cardiovascular risk factors; age, cholesterol, obesity and hypertension are related to diabetes development [24] but, when adjusting for these factors, diabetes in itself is still found to constitute a cardiovascular risk factor [8].

The data relating to the associated cardiovascular risk factors would suggest that a higher incidence of cardiovascular disease could be expected in both groups of diabetics. Others [25] too, have noted that in persons with arteriosclerotic heart disease, without known diabetes, minor abnormalities of carbohydrate metabolism are frequently found.
Since the data from our initial survey [10] and the subsequent surveys $[26,27]$ have been classified into several categories according to whether the diagnosis was based on high fasting blood glucose values, or according to varying degrees of GTT abnormality, we should be able to examine in greater detail the hypothesis of increasing vascular complications with increasing abnormality of the GTT.

The difference in angina pectoris and sudden death experience of previously diagnosed and newly diagnosed diabetics requires further investigation and confirmation. The data would support a suggestion $[28,13]$ that the usual mechanisms producing angina pectoris and sudden death differ from those resulting in myocardial infarction. Angina and sudden death may be acute ischaemic phenomena independent of actual occlusion, while myocardial infarction usually requires actual occlusion and is not precipitated by ischaemia alone. Suggestive support of our data relating to a lower than expected incidence of angina pectoris in newly diagnosed diabetics is to be found in the observation of Root et al. [29], based on clinical evidence, that angina pectoris occurs a decade later in diabetics than in nondiabetic patients and its frequency trebles during the second decade of diabetes. The explanation for the difference in angina pectoris experience might be found in the pathophysiology of cardiac pain, for example a close correlation has been shown between the symptoms of angina pectoris and the excessive regional formation of intermediate products of carbohydrate metabolism [30].

Acknowledgements. This study was a collaborative project between the National Heart and Lung Institute, Bethesda, Maryland, U.S. A., the Hadassah Medical Organization and the Ministry of Health, Jerusalem, Israel, and was supported by PL 480 counterpart funds, Research Agreement No. 375106.

\section{References}

1. Bryfogle, J. W., Bradley, R.F.: The vascular complications of diabetes mellitus. A clinical study. Diabetes 6, 159-167 (1957)

2. Liebow, I. M., Hellerstein, H.K., Miller, M.: Arteriosclerotic heart disease in diabetes mellitus (a clinical study of $383 \mathrm{pa-}$ tients). Am. J. Med. 18, 438-447 (1955)

3. Marble, A.: Coronary artery disease in the diabetic. Diabetes 4, 290-295 (1955)

4. Bell, E.T.: A postmortem study of vascular disease in diabetics. Arch. Pathol. 53, 444 (1952)

5. Kessler, I.I.: Mortality experience of diabetic patients. A twenty six year follow-up study. Am. J. Med. 51, 715-724 (1971)

6. Ostrander, L. D., Francis, T., Hayner, N. S., Kjelsberg, M. O., Epstein, F.H.: The relationship of cardiovascular disease to hyperglycaemia. Ann. Intern. Med. 62, 1188-1198 (1965)

7. Garcia, M.J., McNamara, P.M., Gordon, T., Kannell, W. B.: Morbidity and mortality in diabetics in the Framingham population. Diabetes 23, 105-111 (1974) 
8. Goldbourt, U., Medalie, J.H., Neufeld, H.N.: Clinical myocardial infarction over a five-year period. III. A multivariate analysis of incidence, the Israel Ischaemic Heart Disease Study. J. Chronic Dis. 28, 217-237 (1975)

9. Groen, J.J., Medalie, J.H., Neufeld, H.N., Riss, E., Bachrach, C. A., Mount, F.W., Smith, H.: An epidemiologic investigation of hypertension and ischaemic heart disease within a defined segment of the adult male population of Israel. Isr. J. Med. Sci. 4, 177-194 (1968)

10. Herman, J.B., Mount, F.W., Medalie, J.H., Groen, J.J., Dublin, T.D., Neufeld, N.H., Riss, E.: Diabetes prevalence and serum uric acid. Observations among 10000 men in a survey of ischaemic heart disease in Israel. Diabetes 16, 858-868 (1967)

11. Medalie, J.H., Neufeld, H.N., Riss, E., Groen, J. J., Kahn, H. A., Bachrach, C. A.: Variations in prevalence of ischaemic heart disease in defined segments of the male population in Israel. Isr. J. Med. Sci. 4, 775-788 (1968)

12. World Health Organization criteria of angina pectoris, hypertension and coronary heart disease: classification and criteria for epidemiological studies. WHO Technical Report Series 168, Geneva, 1959

13. Medalie, J. H., Snyder, M., Groen, J. J., Neufeld, H. N., Goldbourt, U., Riss, E.: Angina pectoris among 10000 men. 5 year incidence and univariate analysis. Am. J. Med. 55, 583-594 (1973)

14. Medalie, J.H., Kahn, H. A., Neufeld, H.N., Riss, E., Goldbourt, U., Perlstein, T., Oron, D.: Myocardial infarction over a five-year period. 1. prevalence, incidence and mortality experience. J. Chronic Dis. 26, 63-84 (1973)

15. Snedecor, G. W., Cochran, W. G.: Statistical methods, sixth edition, p. 221. Ames, Iowa: Iowa State University Press, 1967

16. Keen, H., Rose, G., Pyke, D. A., Boyns, D., Chlouverakis, C., Mistry, S.: Blood-sugar and arterial disease. Lancet 1965 II, $505-508$

17. Balme, H.W., Cole, L.: The heredity of hypertension in diabetes mellitus. Q. J. Med. 20, 335-351 (1951)

18. Pell, S., D'Alonzo, C. A.: Some aspects of hypertension in diabetes mellitus. J. A. M. A. 202, 10-16 (1967)

19. Freedman, R., Moulton, R., Spencer, A.G.: Hypertension and diabetes mellitus. Q. J. Med. 27, 293-305 (1958)

20. Andres, R.: Relation of physiologic changes in aging to medi- cal changes of disease in the aged. Mayo Clin. Proc. 42, 674-684 (1967)

21. Kannel, W. B., Castelli, W.P., Gordon, T., McNamara, P. M.: Serum cholesterol, lipoproteins and the risk of coronary heart disease. The Framingham Study, Ann. Intern. Med. 74, 1-12 (1971)

22. Herman, J.B., Medalie, J.H., Goldbourt, U.: Diabetes prediabetes and uricaemia. Diabetologia 12, 47-52 (1976)

23. Conn, H. L., Horwitz, O. (Eds.): Cardiac and Vascular Diseases p. 1120. Philadelphia: Lea and Febiger 1971

24. Medalie, J.H., Papier, C.M., Goldbourt, U., Herman, J.B.: Major factors in the development of diabetes mellitus in 10000 men. Arch. Intern. Med. 135, 811-817 (1975)

25. Reaven, G., Calciano, A., Cody, R., Lucas, C., Miller, R. Carbohydrate intolerance and hyperlipidaemia in patients with myocardial infarction without known diabetes mellitus. J. Clin. Endocrinol. 23, 1013-1023 (1963)

26. Herman, J.B., Medalie, J.H., Kahn, H.A., Neufeld, H.N., Riss, E., Perlstein, T.: Diabetes incidence. A two-year followup of 10000 men in a survey of ischaemic heart disease in Israel. Diabetes 19, 938-943 (1970)

27. Medalie, J.H., Papier, C., Herman, J.B., Goldbourt, U., Tamir, S., Neufeld, H.N., Riss, E.: Diabetes mellitus among 10000 adult men 1 . Five year incidence and associated variables. Isr. J. Med. Sci. 10, 681-697 (1974)

28. Kannel, W.B., Le Bauer, E. J., Dawber, T.R., McNamara, P. M.: Relation of body weight to development of coronary heart disease. The Framingham Study. Circulation 35, 734-744 (1967)

29. Root, H. R., Sharkey, P.: Coronary arteriosclerosis in diabetes mellitus. N. Engl. J. Med. 215, 605-612 (1936)

30. Opie, L.H.: Metabolism of the heart in health and disease. Am. Heart J. 76, 685-698 (1968)

Received: October 15, 1976, and in revised form:

January 14, 1977

Dr. J. B. Herman

Department Medicine C

Hadassah Hebrew University Hospital

Jerusalem

Israel 